\title{
Optimal Regulation of Impulsive Fractional Differential Equation with Delay and Application to Nonlinear Fractional Heat Equation
}

\author{
Wichai Witayakiattilerd ${ }^{1}$ \\ ${ }^{1}$ Department of Mathematics, King Mongkut's Institute of Technology Ladkrabang, Bangkok, Thailand \\ Correspondence: Wichai Witayakiattilerd, Department of Mathematics, King Mongkut's Institute of Technology \\ Ladkrabang, Bangkok, Thailand. E-mail: kwwichai@kmitl.ac.th
}

Received: March 17, 2013 Accepted: April 26, 2013 Online Published: May 10, 2013

doi:10.5539/jmr.v5n2p94 URL: http://dx.doi.org/10.5539/jmr.v5n2p94

\begin{abstract}
The purpose of this paper was considering the impulsive fractional differential system with time delay. We investigated the existence of solution corresponding to the regulator in the admissible regulator set describing by the compact semigroup on Banach space. The result was applied to nonlinear fractional heat equation.
\end{abstract}

Keywords: fractional differential equation, delay system, optimal control, nonlinear fractional heat equation

\section{Introduction}

There are many paper concerned with fractional differential equation and impulsive fractional differential equations. Such as, in 2008, Gastao, S. F., Frederico has solved the optimization regulation problem in the meaning of Caputo and the fractional Neether's theorem. In 2010, Zhongli Wei with the party have studied on the initial value problems for fractional differential equations in the sense of Riemann-Liouville fractional derivative. These papers are motivation of this research.

For this article, we discuss the nonlinear-retarded functional impulsive fractional differential equations

$$
\left\{\begin{array}{l}
D_{t}^{\alpha} x(t)=A x(t)+f(t, x(t), x(t-l), \ldots, x(t-m l))+\mathcal{B}(t) u(t), \quad t \in I \backslash \mathcal{D} \\
\Delta x\left(t_{i}\right)=J_{i}\left(x\left(t_{i}\right)\right), \quad t_{i} \in \mathcal{D} \\
x(0)=x_{0}, \quad x(t)=\varphi(t), \quad t \in[-m l, 0)
\end{array}\right.
$$

for some positive integer $m$ and positive real $l$ where $I=[0, T], \mathcal{D}=\left\{t_{1}, t_{2}, \ldots, t_{n}\right\}, A$ is a generator of a semigroup $\{S(t)\}_{t \geq 0}$ on Banach space (B-space) $\mathcal{X}$ with some conditions, $\Delta x\left(t_{i}\right)=x\left(t_{i}^{+}\right)-x\left(t_{i}\right)$ denote the incitement of state $x$ at time $t_{i}$ with the magnitude of incitement $J_{i}, i=1,2, \ldots, n$. Then, we study a minimizing regulation problem of the system with the objective functional $\mathcal{P}$, that is, to search $u_{0}$ an element of a family of admissible regulators $\mathcal{A}_{a d}$ which

$$
\mathcal{P}\left(u_{0}\right) \leq \mathcal{P}(u), \text { for all } u \in \mathcal{A}_{a d} .
$$

In this case, define the objective functional by $\mathcal{P}(u)=\int_{0}^{T} r\left(t, x(t), x_{t}, u(t)\right) d t+g(x(T))$ for all $u \in \mathcal{A}_{a d}$ with a given running function $r$ and a given terminal function $g$ and $x$ is a solution of the system consistent with a regulator $u \in \mathcal{A}_{a d}$.

The extent of this work starts with background, some important descriptions and theorems for substantiating the main resultant of the paper, for example, definition of fractional derivative, some fractional integral inequalities and the generalized of the Ascoli-Arzela Theorem (AAT) are recommended. The investigation of existence of solution for the system is then presented in Subsection 3.1. Furthermore, the optimization regulation problem is solved and written in Subsection 3.2. In the last section (Section 4), we solve the minimization regulation problem of nonlinear fractional heat by using our main result.

\section{Background}

Let $X$ and $Y$ be any B-spaces. Throughout this article, the symbol, $\mathcal{L}(X, Y)$ represent the space of bounded linear operators from $\mathcal{X}$ to $Y$. Especially, $\mathcal{L}(\mathcal{X})=\mathcal{L}(X, \mathcal{X})$ which norm $\|\cdot\|_{\mathcal{L}(X)}$. The symbol, $P C([a, b], \mathcal{X})$ represent the 
B-spaces of piecewise continuous functions from $[a, b]$ to $\mathcal{X}$ with the supremum norm $\|\cdot\|_{P C([a, b], X)}$. The interval $[0, T]$ is denoted by $I$.

\subsection{Description of Fractional Calculus}

Definition 1 Let $f \in C(\mathfrak{R}, \mathfrak{R})$ and $\alpha>0$. Define the fractional derivative of function $f$ by

$$
D^{-\alpha} f(t) \equiv \frac{1}{\Gamma(\alpha)} \int_{0}^{t} f(s)(t-s)^{\alpha-1} d s
$$

for positive,

$$
D^{\alpha} f(t)=D^{n}\left(D^{\alpha-n} f(t)\right)
$$

which $0<n-\alpha<1, D^{n}$ denote the ordinary derivative of order integer $n$ and $\Gamma(\cdot)$ denote the Gamma function.

Lemma 2 (Jumarie, G.) Given $0<\alpha \leq 1$. Let $f, g$ and $u$ be the $\alpha-$ th differentiable functions. Then theses equalities hold;

$$
\begin{gathered}
D^{\alpha}[f(t) g(t)]=f(t) D^{\alpha} g(t)+f(t) D^{\alpha} g(t), \\
D^{\alpha} f(u(t))=D_{u}^{\alpha} f(u)\left(\frac{d u}{d t}\right)^{\alpha} .
\end{gathered}
$$

Theorem 3 Let $\varphi \in C([-r, 0], \mathcal{X})$. Suppose that $\mathcal{G} \subseteq\{x \in P C([-r, T], \mathcal{X}) \mid x(t)=\varphi(t)$ for $t \in[-r, 0]\}$. If these conditions hold:

1) $\mathcal{G}$ is a uniformly bounded subset of $P C(I, X)$

2) $\mathcal{G}$ is equicontinuous in $\left(0, t_{1}\right),\left(t_{1}, t_{2}\right), \ldots,\left(t_{n}, T\right)$

3) Its $t$-section, $\mathcal{G}(t) \equiv\left\{x(t) \mid x \in \mathcal{G}, t \in[-r, T] \backslash\left\{0, t_{1}, \ldots, t_{n}, T\right\}\right\}, \mathcal{G}\left(t^{+}\right) \equiv\left\{x\left(t^{+}\right) \mid x \in \mathcal{G}\right\}$ and $\mathcal{G}\left(t^{-}\right) \equiv\left\{x\left(t^{-}\right) \mid x \in \mathcal{G}\right\}$ are relatively compact $(R C P)$ subsets of $\mathcal{X}$.

Then $\mathcal{G}$ is a $R C P$ subset of $P C([-r, T], \mathcal{X})$.

Proof. Let $\left\{x_{m}\right\}$ be any sequence of $\mathcal{G}$. We have $\left\{\left.x_{m}\right|_{\left[0, t_{1}\right]}\right\} \subset C\left(\left[0, t_{1}\right], \mathcal{X}\right)$. Applying the AAT on interval $\left[0, t_{1}\right]$, there is a subsequence of $\left\{x_{m}\right\}$, labeled by $\left\{x_{m}\right\}$ again, s.t.

$$
\left.x_{m}\right|_{\left[0, t_{1}\right]} \rightarrow x^{1} \text { in } C\left(\left[0, t_{1}\right], \mathcal{X}\right) \text { as } m \rightarrow \infty .
$$

See $\left\{x_{m} \mid\left[t_{1}, t_{2}\right]\right\} \subset C\left(\left[t_{1}, t_{2}\right], \mathcal{X}\right)$ and let $x_{m}\left(t_{1}\right)=x_{m}\left(t_{1}^{+}\right)$. By using the AAT on interval $\left[t_{1}, t_{2}\right],\left\{x_{m} \mid\left[t_{1}, t_{2}\right]\right\}$ is a RCP subset of $C\left(\left[t_{1}, t_{2}\right], \mathcal{X}\right)$. So, there is a subsequence s.t.

$$
\left.x_{m}\right|_{\left[t_{1}, t_{2}\right]} \rightarrow x^{2} \text { in } C\left(\left[t_{1}, t_{2}\right], \mathcal{X}\right) \text { as } m \rightarrow \infty .
$$

Continue this process until time interval $\left[t_{m}, T\right]$. Then there exists a subsequence $\left\{x_{m}\right\}$, s.t.

$$
\left.x_{m}\right|_{\left[t_{n}, T\right]} \rightarrow x^{n+1} \text { in } C\left(\left[t_{n}, T\right], \mathcal{X}\right) \text { as } m \rightarrow \infty .
$$

Define $x(t)=x^{i}(t), t \in\left[t_{i-1}, t_{i}\right]$ for each $i \in\{1, \ldots, n+1\}$. We obtain $x \in P C([-r, T], \mathcal{X})$ and

$$
\left.x_{m}\right|_{\left[t_{n}, T\right]} \rightarrow x \text { in } P C([-r, T], \mathcal{X}) \text { as } m \rightarrow \infty .
$$

Therefore $\mathcal{G}$ is RCP.

\subsection{Impulsive Integral Inequalities}

Throughout this section, the family of all functions map from $\mathfrak{R}^{+}$to $\mathfrak{R}$ such that their derivatives of order $\alpha$ exist on $\mathfrak{R}^{+}-\left\{t_{k}\right\}$ and left continuous at $t_{k}, k \in\{1,2, \ldots, n\}$ for $0<t_{k}<t_{k+1}$ is represented by $P C^{\alpha}\left(\mathfrak{R}^{+}, \mathfrak{R}\right)$.

Theorem 4 Given $0<\alpha \leq 1$. Let $y \in P C^{\alpha}\left(\mathfrak{R}^{+}, \mathfrak{R}\right)$ which respect to,

$$
\begin{gathered}
y^{\alpha}(t) \leq y(t) p(t)+q(t), t \neq t_{k} \\
y\left(t_{k}^{+}\right) \leq a_{k} y\left(t_{k}\right), y(0)=a_{0}
\end{gathered}
$$

where $a_{k}, k=1,2, \ldots$ are non-negative constants and $p, q \in C\left(\mathfrak{R}^{+}, \mathfrak{R}\right)$. Then

$$
y(t) \leq \sum_{0 \leq t_{k} \leq t}\left(\prod_{t_{k} \leq t_{j} \leq t} a_{j} e^{\int_{t_{j}}^{t_{j+1}} \phi_{\alpha-1}\left(t_{k+1}-s\right) p(s) d s}\right) \cdot\left(\int_{t_{k}}^{t_{k+1}} \phi_{\alpha-1}\left(t_{k+1}-s\right) q(s) e^{-\int_{t_{k}}^{s} \phi_{\alpha-1}(s-r) p(r) d r} d s\right)
$$


where $\phi_{\alpha}(t)=\frac{t^{\alpha}}{\Gamma(\alpha+1)}$.

Proof. Let $t \in\left[0, t_{1}\right]$. Then, we get from inequality (7),

$$
D_{t}^{\alpha}\left[y(t) e^{-\int_{0}^{t} \phi_{\alpha-1}(t-s) p(s) d s}\right] \leq q(t) e^{-\int_{0}^{t} \phi_{\alpha-1}(t-s) p(s) d s} .
$$

By integrating of order $\alpha$ from 0 to $t$,

$$
\begin{aligned}
y(t) & \leq e^{\int_{0}^{t} \phi_{\alpha-1}(t-s) p(s) d s}\left[a_{0}+\int_{0}^{t} \phi_{\alpha-1}(t-s) q(s) e^{-\int_{0}^{s} \phi_{\alpha-1}(s-r) p(r) d r} d s\right] \\
& =a_{0} e^{\int_{0}^{t} \phi_{\alpha-1}(t-s) p(s) d s}+e^{\int_{0}^{t} \phi_{\alpha-1}(t-s) p(s) d s} \int_{0}^{t} \phi_{\alpha-1}(t-s) q(s) e^{-\int_{0}^{s} \phi_{\alpha-1}(s-r) p(r) d r} d s .
\end{aligned}
$$

For $\left[t_{1}, t_{2}\right]$, by inequality (7), we get

$$
D^{\alpha}\left[y(t) e^{-\int_{t_{1}}^{t} \phi_{\alpha-1}(t-s) p(s) d s}\right] \leq q(t) e^{-\int_{t_{1}}^{t} \phi_{\alpha-1}(t-s) p(s) d s}
$$

by integrating of order $\alpha$ from $t_{1}$ to $t$,

$$
y(t) \leq y\left(t_{1}^{+}\right) e^{\int_{t_{1}}^{t} \phi_{\alpha-1}(t-s) p(s) d s}+e^{\int_{t_{1}}^{t} \phi_{\alpha-1}(t-s) p(s) d s} \int_{t_{1}}^{t} \phi_{\alpha-1}(t-s) q(s) e^{-\int_{t_{1}}^{s} \phi_{\alpha-1}(s-r) p(r) d r} d s
$$

and from inequality (8), we get

$$
y\left(t_{1}^{+}\right) \leq a_{1} y\left(t_{1}\right) \leq a_{0} a_{1} e^{t_{0}^{t_{1}} \phi_{\alpha-1}\left(t_{1}-s\right) p(s) d s}+a_{1} e^{\int_{0}^{t_{1}} \phi_{\alpha-1}\left(t_{1}-s\right) p(s) d s} \int_{0}^{t_{1}} \phi_{\alpha-1}\left(t_{1}-s\right) q(s) e^{-\int_{0}^{s} \phi_{\alpha-1}(s-r) p(r) d r} d s .
$$

Hence, we obtain for $t \in\left[t_{1}, t_{2}\right]$,

$$
\begin{aligned}
y(t) & \leq a_{0} a_{1} e^{\int_{0}^{t_{1}} \phi_{\alpha-1}\left(t_{1}-s\right) p(s) d s} e^{\int_{t_{1}}^{t} \phi_{\alpha-1}(t-s) p(s) d s} \\
& +a_{1} e^{\int_{0}^{t_{1}} \phi_{\alpha-1}\left(t_{1}-s\right) p(s) d s} e^{\int_{t_{1}}^{t} \phi_{\alpha-1}(t-s) p(s) d s} \int_{0}^{t_{1}} \phi_{\alpha-1}\left(t_{1}-s\right) q(s) e^{-\int_{0}^{s} \phi_{\alpha-1}(s-r) p(r) d r} d s \\
& +e^{\int_{t_{1}}^{t} \phi_{\alpha-1}(t-s) p(s) d s} \int_{t_{1}}^{t} \phi_{\alpha-1}(t-s) q(s) e^{-\int_{t_{1}}^{s} \phi_{\alpha-1}(s-r) p(r) d r} d s .
\end{aligned}
$$

Assume that inequality (9) holds for $t \in\left[0, t_{k}\right]$ some integer $k>1$. Then for $t \in\left[t_{k}, t_{k+1}\right]$, the result of inequality (7) that

$$
D^{\alpha}\left[y(t) e^{-\int_{t_{k}}^{t} \phi_{\alpha-1}(t-s) p(s) d s}\right] \leq q(t) e^{-\int_{t_{k}}^{t} \phi_{\alpha-1}(t-s) p(s) d s} .
$$

Therefore,

$$
y(t) \leq y\left(t_{k}^{+}\right) e^{\int_{t_{k}}^{t} \phi_{\alpha-1}(t-s) p(s) d s}+e^{\int_{t_{k}}^{t} \phi_{\alpha-1}(t-s) p(s) d s} \int_{t_{k}}^{t} \phi_{\alpha-1}(t-s) q(s) e^{-\int_{t_{k}}^{s} \phi_{\alpha-1}(s-r) p(r) d r} d s .
$$

Using inequality (8), we obtain, for $t \in\left[t_{k}, t_{k+1}\right]$,

$$
y(t) \leq a_{k} y\left(t_{k}\right) e^{\int_{t_{k}}^{t} \phi_{\alpha-1}(t-s) p(s) d s}+e^{\int_{t_{k}}^{t} \phi_{\alpha-1}(t-s) p(s) d s} \int_{t_{k}}^{t} \phi_{\alpha-1}(t-s) q(s) e^{-\int_{t_{k}}^{s} \phi_{\alpha-1}(s-r) p(r) d r} d s .
$$

By the induction hypothesis, this can reduced to

$$
y(t) \leq \sum_{0 \leq t_{k} \leq t}\left(\prod_{t_{k} \leq t_{j} \leq t} a_{j} e^{\int_{t_{j}}^{t_{j+1}} \phi_{\alpha-1}\left(t_{k+1}-s\right) p(s) d s}\right)\left(\int_{t_{k}}^{t_{k+1}} \phi_{\alpha-1}\left(t_{k+1}-s\right) q(s) e^{-\int_{t_{k}}^{s} \phi_{\alpha-1}(s-r) p(r) d r} d s\right)
$$

which on simplification give the estimate (7), for $t \in\left[0, t_{k+1}\right]$.

Theorem 5 Let $y \in P C^{\alpha}\left(\mathfrak{R}^{+}, \mathfrak{R}\right), 0<\alpha<1$ which satisfies,

$$
y(t) \leq a+\sum_{0 \leq t_{k} \leq t} \int_{t_{k}}^{t_{k+1}} \phi_{\alpha-1}\left(t_{k+1}-s\right) p(s) y(s) d s+\sum_{0<t_{k}<t} c_{k} y\left(t_{k}\right)
$$


where $a$ and $c_{k} \geq 0$ are constants and $\phi_{\alpha}(t)=\frac{t^{\alpha}}{\Gamma(\alpha+1)}$. Then,

$$
y(t) \leq \prod_{0 \leq t_{k} \leq t}\left(1+c_{k}\right) e^{\int_{t_{k}}^{t_{k}+1} \phi_{\alpha-1}\left(t_{k+1}-s\right) p(s) m(s) d s},
$$

where $c_{0}=a-1$.

Proof. Setting the right hand side equal to $\mu(t)$. Then we have,

$$
\left\{\begin{array}{l}
\mu^{\alpha}(t)=p(t) y(t) ; \quad t \neq t_{k} \\
\mu\left(t_{k}^{+}\right)=\mu\left(t_{k}\right)+c_{k} y\left(t_{k}\right), \quad \mu(0)=a .
\end{array}\right.
$$

Since $y(t) \leq \mu(t)$,

$$
\left\{\begin{array}{l}
\mu^{\alpha}(t)=p(t) \mu(t) ; \quad t \neq t_{k} \\
\mu\left(t_{k}^{+}\right)=\left(1+c_{k}\right) \mu\left(t_{k}\right), \quad \mu(0)=a=c_{0}+1
\end{array}\right.
$$

Applying Theorem 4, we obtain

$$
y(t) \leq \prod_{0 \leq t_{k} \leq t}\left(1+c_{k}\right) e^{\int_{t_{k}}^{t_{k}+1} \phi_{\alpha-1}\left(t_{k+1}-s\right) p(s) d s} .
$$

\section{Main Results}

Let us consider system (1) with assumptions A1-A5;

A1) $f: I \times X^{m+1} \rightarrow \mathcal{X}$ is uniformly continuous in $t$ and locally Lipchitz in $x_{1}, \ldots, x_{m+1}$, that is, for any $\delta>0$, there is some positive $a(\delta)$ s.t.

$$
\left\|f\left(t, x_{1}, \ldots, x_{m+1}\right)-f\left(s, y_{1}, \ldots, y_{m+1}\right)\right\| \leq a(\delta)\left[\left\|x_{1}-y_{1}\right\|+\ldots+\left\|x_{m+1}-y_{m+1}\right\|+|t-s|\right]
$$

provided $\left\|x_{1}-y_{1}\right\|, \ldots,\left\|x_{m+1}-y_{m+1}\right\| \leq \delta$ and for all $s, t \in I$.

A2) there is some constant $c$ s.t.

$$
f\left(t, x_{1}, \ldots, x_{m+1}\right) \leq c\left(1+\left\|x_{1}\right\|+\ldots+\left\|x_{m+1}\right\|\right)
$$

for all $\left(x_{1}, \ldots, x_{m+1}\right) \in \mathcal{X}^{m+1}$.

A3) $Y$ is another separable reflexive B-space that regulator $u$ take the value, that is, $\mathcal{B}(s) \in \mathcal{L}\left(\mathcal{L}_{q}(I, Y), \mathcal{L}_{p}(I, \mathcal{X})\right)$ for all $s \in I$.

A4) $J_{i}: \mathcal{X} \rightarrow \mathcal{X}$ is an operator s.t. $J_{i}(\mathcal{X})$ is a bounded subset of $\mathcal{X}$ which there are $e_{i}>0, i=1,2, \ldots, n$ s.t.

$$
\left\|J_{i}\left(x_{1}(t)\right)-J_{i}\left(x_{2}\right)(t)\right\| \leq e_{i}\left\|x_{1}(t)-x_{2}(t)\right\|,
$$

for all $x_{1}, x_{2} \in X$ and $t \in I$.

A5) $A$ is the infinitesimal generator of a compact semigroup $\{S(t)\}_{t \geq 0}$ such there exist $M \geq 1, \omega>0$ that $\|S(t)\| \leq M e^{\omega t}$ for all $t \geq 0$.

\subsection{Existence of Solution of Impulsive Fractional Differential System}

Definition 6 For any $u \in \mathcal{A}_{a d}$, a function $x \in P C([-m l, T], \mathcal{X})$ is called a $P C$-mild solution corresponding to a regulator $u$ if it satisfies,

$$
\left\{\begin{aligned}
x(t) & =S(\phi(t)) \varphi(0)+\frac{1}{\Gamma(\alpha)} \sum_{0 \leq t_{k} \leq t} \int_{t_{k}}^{t_{k}+1}\left(t_{k+1}-s\right)^{\alpha-1} S(\phi(t)-\phi(s))[f(s, x(s), x(s-l), \ldots, x(s-m l)) \\
& +\mathcal{B}(s) u(s)] d s+\sum_{0<t_{k}<t} S\left(\phi(t)-\phi\left(t_{k}\right)\right) J_{k} x\left(t_{k}\right), \quad t \in I \\
x(t) & =\varphi(t), \quad t \in[-m l, 0]
\end{aligned}\right.
$$


With assumptions A1-A5, the existence of solution of system (1) is proved by using the LSFPT and the compactness of semigroup $\{S(t)\}_{t \geq 0}$. From the Definition 6 , define the operator $F$ by

$$
\left\{\begin{aligned}
F x(t) & =S(\phi(t)) \varphi(0)+\frac{1}{\Gamma(\alpha)} \sum_{0 \leq t_{k} \leq t} \int_{t_{k}}^{t_{k}+1}\left(t_{k+1}-s\right)^{\alpha-1} S(\phi(t)-\phi(s))[f(s, x(s), x(s-l), \ldots, x(s-m l)) \\
& +\mathcal{B}(s) u(s)] d s+\sum_{0<t_{k}<t} S\left(\phi(t)-\phi\left(t_{k}\right)\right) J_{k} x\left(t_{k}\right), \quad t \in I ; \\
F x(t) & =\varphi(t), \quad t \in[-m l, 0] .
\end{aligned}\right.
$$

for all $x \in P C([-m l, T], \mathcal{X})$. Then $F$ is well-defined.

Let $x \in P C([-m l, T], \mathcal{X})$. By using the continuity of $\|x(t)\|$ and $\|x(t-m l)\|$ and assumption $\mathrm{A} 2$, there is some positive constant $N$ s.t.

$$
\|f(s, x(s), x(s-l), \ldots, x(s-k l))\| \leq N \quad \text { for every } s \in I .
$$

Therefore,

$$
\begin{aligned}
\|F x(t)\| & \leq M e^{\omega \phi(t)}\|\varphi\|_{C}+\frac{M e^{\omega \phi(t)}}{\Gamma(\alpha)} \sum_{0 \leq t_{k} \leq t} \int_{t_{k}}^{t_{k+1}}\left(t_{k+1}-s\right)^{\alpha-1}[\|f(s, x(s), x(s-l), \ldots, x(s-m l))\|+\|\mathcal{B}(s) u(s)\|] d s \\
& +M e^{\omega \phi(t)} \sum_{0<t_{k}<t}\left\|J_{k} x\left(t_{k}\right)\right\| \\
& \leq M e^{\omega \phi(t)}\|\varphi\|_{C}+\frac{M N e^{\omega \phi(t)}}{\Gamma(\alpha)} \sum_{0 \leq t_{k} \leq t} \int_{t_{k}}^{t_{k+1}}\left(t_{k+1}-s\right)^{\alpha-1} d s \\
& +\frac{M e^{\omega \phi(t)}}{\Gamma(\alpha)} \sum_{0 \leq t_{k} \leq t} \int_{t_{k}}^{t_{k+1}}\left(t_{k+1}-s\right)^{\alpha-1}\|\mathcal{B}(s) u(s)\| d s+M e^{\omega \phi(t)} \sum_{0<t_{k}<t} e_{k}\left\|x\left(t_{k}\right)\right\| \\
& \leq M e^{\omega \phi(t)}\|\varphi\|_{C}+\frac{M N(n+1) e^{\omega \phi(t)} T^{\alpha}}{\alpha \Gamma(\alpha)}+\frac{M e^{\omega \phi(t)} K}{\Gamma(\alpha)} \sum_{k=0}^{n}\left[\int_{t_{k}}^{t_{k+1}}\left(t_{k+1}-s\right)^{\frac{p(\alpha-1)}{p-1}} d s\right]^{\frac{p-1}{p}}\left[\int_{t_{k}}^{t_{k+1}}\|\mathcal{B}(s) u(s)\|^{p} d s\right]^{\frac{1}{p}} \\
& +M N e^{\omega \phi(t)} \sum_{0<t_{k}<t} e_{k} \\
& \leq M e^{\omega \phi(t)}\|\varphi\|_{C}+\frac{M N e^{\omega \phi(t)} T^{\alpha}}{\alpha \Gamma(\alpha)}+\frac{M e^{\omega \phi(t)}(p-1)(n+1) T^{\frac{p \alpha-1}{p-1}}\|\mathcal{B}(\cdot) u\|_{L_{p}(I, X)}}{(p \alpha-1) \Gamma(\alpha)}+M N e^{\omega \phi(t)} \sum_{k=1}^{n} e_{k}<\infty .
\end{aligned}
$$

Therefore, the operator $F$ is bounded.

Lemma 7 Assume that assumption A1-A5 hold. Then the bounded operator $F$ is continuous.

Proof. Suppose that $x_{n}$ is a sequence in $P C([-m l, T], \mathcal{X})$ converging to $x$ in $P C([-m l, T], \mathcal{X})$. Then there is $N_{0}>0$ and for every $n>N_{0},\left\|x_{n}-x\right\|_{P C} \leq 1$. Then $\left\|x_{n}\right\| \leq 1+\|x\| \equiv \rho$. By using A1, for $s \in(0, T)$ there is $b(\rho)>0$ s.t.

$$
\left\|f\left(s, x_{n}(s), x_{n}(s-l), \ldots, x_{n}(s-m l)\right)-f(s, x(s), x(s-l), \ldots, x(s-m l))\right\| \leq b(\rho)\left\|x_{n}-x\right\|_{P C}
$$

Therefore, we have

$$
\begin{aligned}
\left|F x_{n}(t)-F x(t)\right| \leq & \frac{1}{\Gamma(\alpha)} \sum_{0 \leq t_{k} \leq t} \int_{t_{k}}^{t_{k+1}}\left(t_{k+1}-s\right)^{\alpha-1}\|S(\phi(t)-\phi(s))\| \\
& \cdot\left\|f\left(s, x_{n}(s), x_{n}(s-l), \ldots, x_{n}(s-m l)\right)-f(s, x(s), x(s-l), \ldots, x(s-m l))\right\| d s \\
& +\sum_{0<t_{k}<t}\left\|S\left(\phi(t)-\phi\left(t_{k}\right)\right)\right\|\left\|J_{k} x_{n}\left(t_{k}\right)-J_{k} x\left(t_{k}\right)\right\| \\
& \leq \frac{M e^{\omega T}}{\Gamma(\alpha)} b(\rho)\left\|x_{n}-x\right\|_{P C} \sum_{0 \leq t_{k} \leq t} \int_{t_{k}}^{t_{k+1}}\left(t_{k+1}-s\right)^{\alpha-1} d s+M e^{\omega T} \sum_{0<t_{k}<t} e_{k}\left\|x_{n}\left(t_{k}\right)-x\left(t_{k}\right)\right\| \\
& \leq \frac{M e^{\omega T}}{\alpha \Gamma(\alpha)} b(\rho)\left\|x_{n}-x\right\|_{P C}(n+1) T^{\alpha}+\sum_{k=0}^{k=n} e_{k}\left\|x_{n}-x\right\|_{P C} .
\end{aligned}
$$


Since $\left\|x_{n}-x\right\|_{P C} \rightarrow 0$ as $n \rightarrow+\infty$, so $\left\|F x_{n}-F x\right\| \rightarrow 0$ as $n \rightarrow+\infty$. This implies that $F$ is continuous on $P C([-m l, T], \mathcal{X})$.

Corollary 8 Assume that assumption A1-A5 hold. The operator F maps bounded set into itself.

Proof. The proof is followed from the proof of Lemma 7. For each $r>0$, there is some $\delta>0$ s.t. for every $x \in B_{\delta} \equiv\left\{x \in P C([-r, T], \mathcal{X}) \mid\|x\|_{P C} \leq \delta\right\}$, we have $\|F x\|_{P C} \leq \delta$.

Lemma 9 Suppose assumptions A1-A5 hold. Then the $F$ is operator compact.

Proof. Given a bounded subset $B$ of $P C([-m l, T], \mathcal{X})$. By Corollary $8, F(B)$ is bounded. Define

$$
Q=F(B) \text { and } Q(t)=\{F x(t) \mid x \in B\} .
$$

Clearly, for $t \in[-m l, 0], Q(t)=\{\varphi(t)\}$ is compact. We only necessary consider for $t>0$. Given $\varepsilon>0$. For $0<\varepsilon \leq t \leq T$, for short we denote $f(s, x(s), x(s-l), \ldots, x(s-m l))+\mathcal{B}(s) u(s))$ by $\tilde{f}_{u}(s, x(s))$ where $x=\left(x_{1}, \ldots, x_{m+1}\right)$ which $x_{1}(s)=x(s-l), \ldots, x_{m}(s)=x(s-m l)$ and $x_{m+1}(s)=x(s)$. Define

$$
\begin{aligned}
Q_{\varepsilon}(t) & \equiv F_{\varepsilon}(B)(t) \\
& =S(\phi(\varepsilon)) S(\phi(t)-\phi(\varepsilon)) \varphi_{0}+\frac{S(\phi(\varepsilon))}{\Gamma(\alpha)} \sum_{0 \leq t_{k}<t} \int_{t_{k}}^{t_{k+1}}\left(t_{k+1}-s\right)^{\alpha-1} S\left(\phi\left(t_{k+1}\right)-\phi(\varepsilon)-\phi(s)\right) \widetilde{f}_{u}(s, x(s)) d s \\
& +\frac{S(\phi(\varepsilon))}{\Gamma(\alpha)} \int_{t_{k}}^{t-\epsilon}(t-s)^{\alpha-1} S(\phi(t)-\phi(\varepsilon)-\phi(s)) \widetilde{f}_{u}(s, \underset{-}{x}(s)) d s+S(\phi(\varepsilon)) \sum_{0<t_{k}<t} S\left(\phi\left(t_{k}\right)-\phi(\varepsilon)-\phi(s)\right) J_{k}\left(x\left(t_{k}\right)\right) .
\end{aligned}
$$

By continuity of $\phi(t)$ and compactness of $S(t)$, set $\left\{Q_{\varepsilon}(t) \mid x \in B\right\}$ is RCP in $\mathcal{X}$ for every $\varepsilon$ sufficiently small, $t \in[\varepsilon, T]$. For $t \in\left(0, t_{1}\right]$ the Equation (17) reduce to

$$
Q_{\varepsilon}(t)=F_{\varepsilon}(B)(t)=S(\phi(\varepsilon)) S(\phi(t)-\phi(\varepsilon)) \varphi_{0}+\frac{S(\phi(\varepsilon))}{\Gamma(\alpha)} \int_{0}^{t-\varepsilon}(t-s)^{\alpha-1} S(\phi(t)-\phi(\varepsilon)-\phi(s)) \widetilde{f}_{u}(s, \underset{-}{x}(s)) d s .
$$

Furthermore, the continuity of $\|x(t)\|$ and $\|x(t-m l)\|$ on $\left(0, t_{1}\right)$ for all $m=1, \ldots, k$ imply that there is a positive $N$ s.t. $\|x(t)\|,\|x(t-m l)\| \leq N$. By assumption $\mathrm{A} 2$ and assumption $\mathrm{A} 4$, there exist a positive constant $c$ such that

$$
\left\|\widetilde{f}_{u}(s, x(s))\right\| \leq c\left(1+(m+1)\|x\|_{P C}\right)+\|\mathcal{B}(\cdot) u\|_{L_{p}(I, X)} \equiv L_{u} .
$$

Then for $t \in\left[\varepsilon, t_{1}\right]$,

$$
\begin{aligned}
\sup _{x \in B}\left\|F x(t)-F_{\varepsilon} x(t)\right\| & =\frac{1}{\Gamma(\alpha)} \sup _{x \in B} \| \int_{0}^{t}(t-s)^{\alpha-1} S(\phi(t)-\phi(s)) \widetilde{f}_{u}(s, x(s)) d s \\
& -S(\phi(\varepsilon)) \int_{0}^{t-\varepsilon}(t-s)^{\alpha-1} S(\phi(t)-\phi(\varepsilon)-\phi(s)) \widetilde{f}_{u}(s, \underset{-}{x}(s)) d s \| \\
& =\frac{1}{\Gamma(\alpha)} \sup _{x \in B}\left\|\int_{t-\varepsilon}^{t}(t-s)^{\alpha-1} S(\phi(t)-\phi(s)) \widetilde{f}_{u}(s, x(s)) d s\right\| \\
& \leq \frac{M L_{u}}{\Gamma(\alpha)} \int_{t-\varepsilon}^{t}(t-s)^{\alpha-1} d s=\frac{M L_{u} \varepsilon^{\alpha}}{\Gamma(\alpha+1)}
\end{aligned}
$$

Hence, there exist RCP sets arbitrary close to the set $Q(t)$ for $t \in\left[0, t_{1}\right]$. Therefore, $Q(t)$ itself is RCP in $\mathcal{X}$ on the interval $\left[0, t_{1}\right]$. On the interval $\left(t_{1}, t_{2}\right]$, we define

$$
Q\left(t_{1}^{+}\right) \equiv Q\left(t_{1}^{-}\right)+J_{1}\left(Q\left(t_{1}^{-}\right)\right)=Q\left(t_{1}\right)+J_{1}\left(Q\left(t_{1}\right)\right) .
$$

By the condition A3, we get $J_{1}\left(Q\left(t_{1}\right)\right)$ is RCP and this implies $Q\left(t_{1}^{+}\right)$is also RCP. Let $x\left(t_{1}^{+}\right)=x_{1}$. Then for $t \in\left(t_{1}\right.$, $\left.t_{2}\right]$, the Equation (17) reduce to

$$
\begin{aligned}
Q_{\varepsilon}(t) & =F_{\varepsilon}(B)(t) \\
& =S(\phi(\varepsilon)) S\left(\phi(t)-\phi\left(t_{1}\right)-\phi(\varepsilon)\right) x_{1}+\frac{S(\phi(\varepsilon))}{\Gamma(\alpha)} \int_{0}^{t_{k+1}}\left(t_{1}-s\right)^{\alpha-1} S\left(\phi\left(t_{1}\right)-\phi(\varepsilon)-\phi(s)\right) \widetilde{f}_{u}(s, x(s)) d s \\
& +\frac{S(\phi(\varepsilon))}{\Gamma(\alpha)} \int_{t_{1}}^{t-\varepsilon}(t-s)^{\alpha-1} S(\phi(t)-\phi(\varepsilon)-\phi(s)) \widetilde{f}_{u}(s, \underset{-}{x}(s)) d s+S(\phi(\varepsilon)) S\left(\phi\left(t_{1}\right)-\phi(\varepsilon)-\phi(s)\right) J_{1}\left(x\left(t_{1}\right)\right) .
\end{aligned}
$$


Furthermore, for $t \in\left[t_{1}+\varepsilon, t_{2}\right]$,

$$
\sup _{x \in B}\{\|F x(t)-F x(t)\|\} \leq \frac{M L_{u} \varepsilon^{\alpha}}{\Gamma(\alpha+1)} .
$$

Therefore $Q(t)$ is RCP on $\left[t_{1}, t_{2}\right]$.

Generally, given any $t_{k} \in \mathcal{D}=\left\{t_{0}=0, t_{1}, t_{2}, \ldots, t_{n}, t_{n+1}=T\right\}$, define $x\left(t_{k}^{+}\right)=x_{k}$ and

$$
Q\left(t_{k}^{+}\right) \equiv Q\left(t_{k}^{+}\right)+J_{k}\left(Q\left(t_{k}^{-}\right)\right)=Q\left(t_{k}\right)+J_{k}\left(Q\left(t_{k}\right)\right) \text { for } t_{k} \in \mathcal{D} \text {. }
$$

Similarly, for $t \in\left(t_{k}, t_{k+1}\right]$, the Equation (17) reduce to

$$
\begin{aligned}
Q_{\varepsilon}(t) & =F_{\varepsilon}(B)(t) \\
& =S(\phi(\varepsilon)) S\left(\phi(t)-\phi\left(t_{k}\right)-\phi(\varepsilon)\right) x_{k}+\frac{S(\phi(\varepsilon))}{\Gamma(\alpha)} \sum_{0 \leq t_{k}<t} \int_{t_{k}}^{t_{k+1}}\left(t_{k+1}-s\right)^{\alpha-1} S\left(\phi\left(t_{k+1}\right)-\phi(\varepsilon)-\phi(s)\right) \widetilde{f}_{u}(s, \underset{-}{x(s)) d s} \\
& +\frac{S(\phi(\varepsilon))}{\Gamma(\alpha)} \int_{t_{k}}^{t-\varepsilon}(t-s)^{\alpha-1} S(\phi(t)-\phi(\varepsilon)-\phi(s)) \widetilde{f}_{u}(s, \underset{-}{x}(s)) d s+S(\phi(\varepsilon)) \sum_{0<t_{k}<t} S\left(\phi\left(t_{k}\right)-\phi(\varepsilon)-\phi(s)\right) J_{k}\left(x\left(t_{k}\right)\right) .
\end{aligned}
$$

Furthermore, for $\left[t_{k}+\varepsilon, t_{k+1}\right]$,

$$
\sup _{x \in B}\{\|F x(t)-F x(t)\|\} \leq \frac{M L_{u} \varepsilon^{\alpha}}{\Gamma(\alpha+1)} .
$$

By repeating these process till the time interval which expanded, $Q(t)$ is $\mathrm{RCP}$ for $t \in[-m l, T] \backslash \mathcal{D}$ and $Q\left(t_{k}^{+}\right)$is $\mathrm{RCP}$ for $t_{k} \in \mathcal{D}$. Next, we will show that $Q$ is equicontinuous on $\left(t_{k}, t_{k+1}\right), k=0,1, \ldots, n$. Since $B$ is bounded and follow from the inequality (18), there is an $L_{u}>0$ such that

$$
\left\|\widetilde{f_{u}}(s, x(s))\right\| \leq L_{u} .
$$

Let $h>0$ and for $0<t<t+h<t_{1}$ and for $x \in B$. Then

$$
\begin{aligned}
& \|F x(t+h)-F x(t)\| \\
& \leq\|S(\phi(t+h)) \varphi(0)-S(\phi(t)) \varphi(0)\|+\| \frac{1}{\Gamma(\alpha)} \int_{0}^{t+h}(t+h-s)^{\alpha-1} S(\phi(t+h)-\phi(s)) \widetilde{f}_{u}(s, x(s)) d s \\
& -\frac{1}{\Gamma(\alpha)} \int_{0}^{t}(t-s)^{\alpha-1} S(\phi(t)-\phi(s)) \widetilde{f}_{u}(s, x(s)) d s \| \\
& \leq\|S(\phi(t))\|\||S(\phi(h))-I\|\|| \varphi\|_{C}+\frac{1}{\Gamma(\alpha)} \int_{t}^{t+h}(t+h-s)^{\alpha-1}\|S(\phi(t+h)-\phi(s))\|\|\| \widetilde{f}_{u}(s, x(s)) \| d s \\
& +\frac{1}{\Gamma(\alpha)} \int_{0}^{t}\|S(\phi(t)-\phi(s))\|\left\|(t-s+h)^{\alpha-1} S(\phi(h))-(t-s)^{\alpha-1} I\right\|\left\|\tilde{f}_{u}(s, x(s))\right\| d s \\
& \leq M e^{\omega \phi(T)}\|\varphi\|_{C}\|S(\phi(h))-I\|+\frac{M e^{\omega \phi(T)}}{\alpha \Gamma(\alpha)} L_{u} h^{\alpha}+\frac{M e^{\omega \phi(T)}}{\Gamma(\alpha)} L_{u} \int_{0}^{t}\left\|(t-s+h)^{\alpha-1} S(h)-(t-s)^{\alpha-1} I\right\| d s .
\end{aligned}
$$

Since $\lim _{h \rightarrow 0}\left\|(t-s+h)^{\alpha-1} S(\phi(h))-(t-s)^{\alpha-1} I\right\|=0$ and $\lim _{h \rightarrow 0}\|S(\phi(h))-I\|=0$, so $\|F x(t+h)-F x(t)\| \rightarrow 0$ as $h \rightarrow 0$. Hence $F$ is equicontinuous on $\left(0, t_{1}\right)$. In general, for $\left(t_{k}, t_{k+1}\right), k=0,1,2, \ldots, n$, for $t_{k}<t<t+h<t_{k+1}$

$$
\begin{aligned}
\|F x(t+h)-F x(t)\| & \leq\|S(\phi(t))\|\|\mid S(\phi(h))-I\|\left\|x_{k}\right\| \\
& +\frac{1}{\Gamma(\alpha)} \int_{t}^{t+h}(t+h-s)^{\alpha-1}\|S(\phi(t+h)-\phi(s))\|\left\|\mid \widetilde{f}_{u}(s, x(s))\right\| d s \\
& +\frac{1}{\Gamma(\alpha)} \int_{0}^{t}\|S(\phi(t)-\phi(s))\|\left\|\mid(t-s+h)^{\alpha-1} S(\phi(h))-(t-s)^{\alpha-1} I\right\|\left\|\widetilde{f}_{u}(s, x(s))\right\| d s \\
& \leq M e^{\omega \phi(T)}\left\|x_{k}\right\|\|S(\phi(h))-I\|+\frac{M e^{\omega \phi(T)}}{\alpha \Gamma(\alpha)} L_{u} h^{\alpha} \\
& +\frac{M e^{\omega \phi(T)}}{\Gamma(\alpha)} L_{u} \int_{0}^{t}\left\|(t-s+h)^{\alpha-1} S(h)-(t-s)^{\alpha-1} I\right\| d s .
\end{aligned}
$$


Using the same idea, we can show that $Q$ is equicontinuous on $\left(t_{k}, t_{k+1}\right)$ where $k=0,1,2, \ldots, n$. Therefore, Theorem 3 implies that $F(B)$ is a RCP subset of $P C([-m l, T], \mathcal{X})$. Further, $F$ is a compact operator.

Lemma 10 The set $\Omega \equiv\{x \in P C([-m l, T], \mathcal{X}) \mid x=\sigma F x, \sigma \in[0,1]\}$ is bounded on $P C([-m l, T], \mathcal{X})$.

Proof. Let $x \in \Omega$. Since $\varphi$ is continuous, there exist $M_{1}>0$

$$
\|x(t)\|=\|\sigma F x(t)\| \leq\|F x(t)\|=\|\varphi(t)\| \leq M_{1} \text { for all } t \in[-r, 0] .
$$

By using assumptions A2, there are $N_{1}, N_{2}>0$ such that for $t \in I$

$$
\|f(t, x(t), x(t-l), \ldots, x(t-m l))\| \leq N_{1}+N_{2}\|x(t)\|
$$

Using assumption A3 and assumption A4, we get,

$$
\begin{aligned}
\|x(t)\| & =\|\sigma F x(t)\| \leq\|F x(t)\| \\
& \leq\|S(\phi(t))\|\|\varphi\|_{C}+\frac{1}{\Gamma(\alpha)} \sum_{0 \leq t_{k} \leq t} \int_{t_{k}}^{t_{k+1}}\left(t_{k+1}-s\right)^{\alpha-1}\|S(\phi(t)-\phi(s))\|\left[\left\|f\left(s, x(s), x_{1}(s-l), \ldots, x(s-m l)\right)\right\|\right. \\
& +\|\mathcal{B}(s) u(s)\|] d s+\sum_{0<t_{k}<t}\left\|S\left(\phi(t)-\phi\left(t_{k}\right)\right)\right\|\left\|\mid J_{k}\left(x\left(t_{k}\right)\right)\right\| \\
& \leq M e^{\omega \phi(T)}\|\varphi\|_{C}+\frac{M N_{1} e^{\omega \phi(T)}}{\Gamma(\alpha)} \sum_{0 \leq t_{k} \leq t} \int_{t_{k}}^{t_{k+1}}\left(t_{k+1}-s\right)^{\alpha-1} d s+\frac{M N_{2} e^{\omega \phi(T)}}{\Gamma(\alpha)} \sum_{0 \leq t_{k} \leq t} \int_{t_{k}}^{t_{k+1}}\left(t_{k+1}-s\right)^{\alpha-1}\|x(s)\| d s \\
& +\frac{M e^{\omega \phi(T)}}{\Gamma(\alpha)} \sum_{0 \leq t_{k} \leq t} \int_{t_{k}}^{t_{k+1}}\left(t_{k+1}-s\right)^{\alpha-1}\|\mathcal{B}(s) u(s)\| d s+M e^{\omega \phi(T)} \sum_{0<t_{k}<t} e_{k}\left\|x\left(t_{k}\right)\right\| \\
& \leq M e^{\omega \phi(T)}\|\varphi\|_{C}+\frac{M N_{1} e^{\omega \phi(T)}}{\alpha \Gamma(\alpha)} T^{\alpha}(n+1)+\frac{M N_{2} e^{\omega \phi(T)}}{\Gamma(\alpha)} \sum_{0 \leq t_{k} \leq t} \int_{t_{k}}^{t_{k+1}}\left(t_{k+1}-s\right)^{\alpha-1}\|x(s)\| d s \\
& +\frac{M e^{\omega \phi(T)}}{\Gamma(\alpha)} \sum_{k=0}^{n}\left[\int_{t_{k}}^{t_{k+1}}\left(t_{k+1}-s\right)^{\frac{p(\alpha-1)}{p-1}} d s\right]^{\frac{p-1}{p}}\left[\int_{t_{k}}^{t_{k+1}}\|\mathcal{B}(s) u(s)\|^{p} d s\right]^{\frac{1}{p}}+M e^{\omega \phi(T)} \sum_{0<t_{k}<t} e_{k}\left\|x\left(t_{k}\right)\right\| \\
& \leq M e^{\omega \phi(T)}\left[\|\varphi\|_{C}+\frac{N_{1} T^{\alpha}(n+1)}{\alpha \Gamma(\alpha)}+\frac{T^{\frac{p \alpha-1}{p-1}}(p-1)\left(n+1\|\mathcal{B}(\cdot) u\|_{L_{p}(I, X)}\right.}{(p \alpha-1) \Gamma(\alpha)}\right] \\
& +\frac{M N_{2} e^{\omega \phi(T)}}{\Gamma(\alpha)} \sum_{0 \leq t_{k} \leq t} \int_{t_{k}}^{t_{k+1}}\left(t_{k+1}-s\right)^{\alpha-1}\|x(s)\| d s+M e^{\omega \omega(T)} \sum_{0<t_{k}<t} e_{k}\left\|x\left(t_{k}\right)\right\| .
\end{aligned}
$$

Using Theorem 5, there is $M_{2}>0$ s.t. $\|x(t)\| \leq M_{2}$, for all $x \in \Omega$. Hence, $\Omega$ is a bounded subset of $P C([-m l, T]$, $X)$.

Theorem 11 Assume that assumptions A1-A5 hold, then the system (1) has at least one PC-mild solution corresponding to a regulator $u \in \mathcal{A}_{a d}$ on $[-m l, T]$.

Proof. Define the operator $F$ as (16). Then by Lemma 7 and Lemma 9, we have $F$ is continuous on $P C([-m l, T], \mathcal{X})$ and compact. Set $\Omega \equiv\{x \in P C([-m l, T], \mathcal{X}) \mid x=\sigma F x, \sigma \in[0,1]\}$. The Lemma 10 implies $\Omega$ is a bounded subset of $P C([-m l, T], \mathcal{X})$. Then, the LSFPT to implies that $F$ has a fixed point in $P C([-m l, T], \mathcal{X})$. Therefore, system (1) has at least $P C$-mild solution corresponding to the regulator $u \in \mathcal{A}_{a d}$ on $P C([-m l, T], \mathcal{X})$.

\subsection{Existence of Optimal Regulation}

Let $\mathcal{A}_{a d}$ be the admissible regulator set. Note that the result in Section 3.1 implies that for each regulator $u \in \mathcal{A}_{a d}$, there exits a PC-mild solution $x$ corresponding to the regulator $u$.

Let us consider the minimization problem $(P)$ corresponding to system (1). Find a regulator $u^{0} \in \mathcal{A}_{a d}$ s.t.

$$
\mathcal{P}\left(u^{0}, x^{0}\right) \leq \mathcal{P}(u, x) \text { for all } u \in \mathcal{A}_{a d}
$$

which $\mathcal{P}(u, x)=\int_{0}^{T} r(t, x(t), x(t-l), \ldots, x(t-m l), u(t)) d t+g(x(T))$ and $x$ is a mild solution of system (1) corresponding to a regulator $u \in \mathcal{A}_{a d}$, the order pair $(u, x)$ is called the admissible pair. For convenience, $\mathcal{P}(u, x)$ is short written by $\mathcal{P}(u)$.

We solve the optimizing regulation problem in this paper under the following assumptions, label by A6-A10; 
A6) $r: I \times X^{m+1} \times Y \rightarrow(-\infty, \infty]$ is Borel measurable.

A7) $r(t, \cdot, \cdot, \cdot)$ is sequentially lower semicontinuous on $X^{m+1} \times Y$ for a.e. on $I$.

A8) $r(t, \xi, \cdot)$ is convex on $Y$ for all $\xi \in X^{m+1}$ and for a.e. $t \in I$.

A9) There exist constants $a_{i}, b>0$ for all $i=1, \ldots, m+1$ and $\eta \in \mathcal{L}_{1}(I, \mathfrak{R})$ s.t.

$$
r(t, \xi, u) \geq \eta(t)+\sum_{i=1}^{m+1} a_{i}\left\|\xi_{1}\right\|+b\|u\|_{Y}^{q}
$$

where $\xi=\left(\xi_{1}, \ldots, \xi_{m+1}\right) \in \mathcal{X}^{m+1}$.

A10) $g: \mathcal{X} \rightarrow \mathfrak{R}$ is nonnegative continuous function.

Theorem 12 Suppose that assumption A1-A10 hold. Then the problem $(P)$ corresponding to system (1) has at least one solution, that is, there is an admissible pair $\left(u^{0}, x^{0}\right)$ such that

$$
\mathcal{P}\left(u^{0}, x^{0}\right) \leq \mathcal{P}(u, x) \text { for all } u \in \mathcal{A}_{a d} .
$$

Proof. If $\inf \left\{\mathcal{P}(u) \mid u \in \mathcal{A}_{a d}\right\}=+\infty$, it is well done. Suppose that $\inf \left\{\mathcal{P}(u) \mid u \in \mathcal{A}_{a d}\right\}=w<+\infty$. By assumption A9, there are constants $a_{i}, b>0$ for all $i=0, \ldots, m$ and $\eta \in \mathcal{L}_{1}(I, \mathfrak{R})$ such that

$$
r(t, x(t), x(t-l), \ldots, x(t-m l), u) \geq \eta(t)+\sum_{i=0}^{m} a_{i}\|x(t-i l)\|+b\|u\|_{Y}^{q} .
$$

Since $\eta$ is non-negative, we get

$$
\mathcal{P}(u) \geq \int_{0}^{T} \eta(t) d t+\sum_{i=0}^{m} a_{i} \int_{0}^{T}\|x(t-i l)\| d t+b \int_{0}^{T}\|u(t)\|_{Y}^{q} d t \geq-\sigma>-\infty .
$$

for some $\sigma>0$, for all $u \in \mathcal{A}_{a d}$. Hence $w \geq-\sigma>-\infty$. By definition of minimum, there exists a minimizing sequence $\left\{u_{n}\right\}$ of $\mathcal{P}$, that is $\lim _{n \rightarrow \infty} \mathcal{P}\left(u_{n}\right)=w$ and

$$
\mathcal{P}\left(u_{n}\right) \geq \int_{0}^{T} \eta(t) d t+\sum_{i=0}^{m} a_{i} \int_{0}^{T}\left\|x_{n}(t-i l)\right\| d t+b \int_{0}^{T}\left\|u_{n}(t)\right\|_{Y}^{q} d t .
$$

So, there exists $N_{0}>0$ such that

$$
w+w_{1} \geq \mathcal{P}\left(u_{n}\right) \geq c \int_{0}^{T}\left\|u_{n}(t)\right\|_{Y}^{q} d t, \text { for all } n \geq N_{0},
$$

for some $w_{1}>0$ and hence $\left\|u_{n}\right\|_{L_{q}(I, Y)}^{q} \leq \frac{w_{1}+w}{c}$. Thus $u_{n}$ is a bounded sequence containing in the reflexive Bspace $\mathcal{L}_{q}(I, Y)$. Therefore, $u_{n}$ has a convergence subsequence, relabelled as $u_{n}$ and $u_{n} \rightarrow u_{0}$ for some $u_{0} \in$ $\mathcal{A}_{a d}=\mathcal{L}_{q}(I, Y)$. Let $x_{n} \in P C([-m l, T], \mathcal{X})$ be a sequence of PC-mild solutions of system (1) corresponding to the regulators sequence $u_{n}$;

$$
\left\{\begin{aligned}
x_{n}(t) & =S(\phi(t)) \varphi(0)+\frac{1}{\Gamma(\alpha)} \sum_{0 \leq t_{k} \leq t} \int_{t_{k}}^{t_{k}+1}\left(t_{k+1}-s\right)^{\alpha-1} S(\phi(t)-\phi(s))\left[f\left(s, x_{n}(s), x_{n}(s-l), \ldots, x_{n}(s-m l)\right)\right. \\
& \left.+\mathcal{B}(s) u_{n}(s)\right] d s+\sum_{0<t_{k}<t} S\left(\phi(t)-\phi\left(t_{k}\right)\right) J_{k} x_{n}\left(t_{k}\right), \quad t \in I \\
x_{n}(t) & =\varphi(t), \quad t \in[-m l, 0] .
\end{aligned}\right.
$$

The a priori estimate implies there is some positive constant $\rho$ s.t.

$$
\left\|x_{n}\right\|_{P C([-r, T], \mathcal{X})} \leq \rho \text { for all } n=0,1,2, \ldots
$$

Let $x^{0}$ be a PC-mild solution of system (1) corresponding to regulator $u^{0}$, i.e.,

$$
\left\{\begin{aligned}
x^{0}(t) & =S(\phi(t)) \varphi(0)+\frac{1}{\Gamma(\alpha)} \sum_{0 \leq t_{k} \leq t} \int_{t_{k}}^{t_{k}+1}\left(t_{k+1}-s\right)^{\alpha-1} S(\phi(t)-\phi(s))\left[f\left(s, x^{0}(s), x^{0}(s-l), \ldots, x^{0}(s-m l)\right)\right. \\
& \left.+\mathcal{B}(s) u^{0}(s)\right] d s+\sum_{0<t_{k}<t} S\left(\phi(t)-\phi\left(t_{k}\right)\right) J_{k} x^{0}\left(t_{k}\right), \quad t \in I \\
x^{0}(t) & =\varphi(t), \quad t \in[-m l, 0] .
\end{aligned}\right.
$$


Given $\rho>0$. By using assumption A1, there exist $b(\rho)>0$ such that for $s \in(0, T)$,

$$
\left\|f\left(s, x_{n}(s), x_{n}(s-l), \ldots, x_{n}(s-m l)\right)-f\left(s, x^{0}(s), x^{0}(s-l), \ldots, x^{0}(s-m l)\right)\right\| \leq b(\rho)\left\|x_{n}-x^{0}\right\|_{P C} .
$$

We use the fact that $x_{n}(s)-x^{0}(s)=0$ for $s \in[-r, 0]$, so we have

$$
\begin{aligned}
\left\|x_{n}(s)-x^{0}(s)\right\| & \leq \frac{b(\rho) M e^{\omega \phi(T)}}{\Gamma(\alpha)} \sum_{0 \leq t_{k} \leq t} \int_{t_{k}}^{t_{k+1}}\left(t_{k+1}-s\right)^{\alpha-1}\left\|x_{n}(s)-x^{0}(s)\right\| d s \\
& +\frac{M e^{\omega \phi(T)}}{\Gamma(\alpha)} \sum_{k=1}^{k=n}\left[\int_{t_{k}}^{t_{k+1}}\left(t_{k+1}-s\right)^{\frac{p \alpha-1}{p-1}} d s\right]^{\frac{p-1}{p}}\left[\int_{t_{k}}^{t_{k+1}}\left\|\mathcal{B}(s) u_{n}(s)-\mathcal{B}(s) u^{0}(s)\right\|^{p} d s\right]^{\frac{1}{p}} \\
& +M e^{\omega \phi(T)} \sum_{0<t_{k}<t} e_{k}\left\|x_{n}\left(t_{k}\right)-x^{0}\left(t_{k}\right)\right\| \\
& \leq \frac{M e^{\omega \phi(T)}(p-1)(n+1) T^{\frac{p \alpha-1}{p-1}}\left\|\mathcal{B}(\cdot) u_{n}-\mathcal{B}(\cdot) u^{0}\right\|_{p}}{(p \alpha-1) \Gamma(\alpha)} \\
& +\frac{b(\rho) M e^{\omega \phi(T)}}{\Gamma(\alpha)} \sum_{0 \leq t_{k} \leq t} \int_{t_{k}}^{t_{k+1}}\left(t_{k+1}-s\right)^{\alpha-1}\left\|x_{n}(s)-x^{0}(s)\right\| d s+M e^{\omega \phi(T)} \sum_{0<t_{k}<t} e_{k}\left\|x_{n}\left(t_{k}\right)-x^{0}\left(t_{k}\right)\right\| .
\end{aligned}
$$

Applying Theorem 5, there exist $M_{1}>0$ independent on $u, n$ and $t$ such that

$$
\left\|x_{n}(t)-x^{0}(t)\right\| \leq M_{1}\left\|\mathcal{B}(\cdot) u_{n}-\mathcal{B}(\cdot) u^{0}\right\|_{\mathcal{L}_{q}(I, Y)} .
$$

The strongly continuous of $\mathcal{B}(\cdot)$ implies $\left\|\mathcal{B}(\cdot) u_{n}-\mathcal{B}(\cdot) u^{0}\right\|_{\mathcal{L}_{q}(I, Y)} \stackrel{s}{\rightarrow} 0$. Consequently, $\left\|x_{n}-x^{0}\right\| \stackrel{s}{\rightarrow} 0$ in $P C([-m l, T], \mathcal{X})$. Let us set $r_{n}(t)=r\left(t, x_{n}(t), x_{n}(t-l), \ldots, x(t-m l), u_{n}(t)\right)$ for all $t \in I$. Then by assumption A6 and assumption A9, $\left\{r_{n}(t)\right\}$ is a sequence of non-negative measurable functions. So, by applying Fatou's Lemma,

$$
\varliminf_{n \rightarrow \infty} \int_{0}^{T} r_{n}(t) d t \geq \int_{0}^{T} \underline{\lim _{n \rightarrow \infty}} r_{n}(t) d t .
$$

By assumption $A 7$,

$$
\begin{aligned}
m & =\lim _{n \rightarrow \infty} \mathcal{P}\left(u_{n}\right) \geq \underset{n \rightarrow \infty}{\lim }\left[\int_{0}^{T} r_{n}(t) d t+\Phi\left(x_{n}(T)\right)\right] \geq \int_{0}^{T} \frac{\lim }{n \rightarrow \infty} r\left(t, x_{n}(t),\left(x_{n}\right)_{t}, u_{n}(t)\right) d t+g\left(x^{0}(T)\right) \\
& \geq r\left(t, x^{0}(t), x_{t}^{0}, u^{0}(t)\right) d t+g\left(x^{0}(T)\right)=\mathcal{P}\left(u^{0}\right) .
\end{aligned}
$$

Therefore, $\mathcal{P}\left(u^{0}\right)=m$.

\section{Optimal Regulation of Nonlinear Fractional Heat Equation}

Let us consider the boundary value problem with delay and regulation;

$$
\begin{gathered}
\frac{\partial^{\alpha} y(x, t)}{\partial t^{\alpha}}=\Delta y(x, t)+f(x, t, y(x, t), y(x, t-l), \ldots, y(x, t-m l))+\int_{\Omega} \mathcal{B}(x, \xi) u(\xi, t) d \xi, \quad(x, t) \in \Omega \times I \backslash \mathcal{D} \\
\Delta y\left(x, t_{k}\right)=J_{k}\left(y\left(x, t_{k}\right)\right), \quad t_{k} \in \mathcal{D} \\
y(x, t)=\varphi(x, t), \quad(x, t) \in \bar{\Omega} \times[-m l, 0] \\
y(x, t)=0, \quad(x, t) \in \partial \Omega \times I
\end{gathered}
$$

where $\mathcal{D}=\left\{t_{1}, t_{2}, \ldots, t_{n}\right\}, \Omega$ is boundary domain of $\mathfrak{R}^{N}, \varphi \in C(\bar{\Omega} \times[-m l, 0]), u \in \mathcal{L}_{q}(\Omega \times I), h \in C\left([-m l, T]^{2}, \mathfrak{R}\right)$ and $B: \bar{\Omega} \times \bar{\Omega} \rightarrow \mathfrak{R}$ is continuous. Suppose that these conditions hold;

HHf) $f: \bar{\Omega} \times I \times \mathfrak{R} \times \mathfrak{R}^{N} \rightarrow \mathfrak{R}$ and there are $L_{1}, L_{2} \geq 0$ s.t.

$$
\begin{gathered}
|f(x, t, \xi, \eta)| \leq L_{1}(1+|\xi|+|\eta|) \text {, and } \\
\left|f(x, t, \xi, \eta)-f\left(x, s, \xi_{1}, \eta_{1}\right)\right| \leq L_{2}\left(|t-s|+\left|\xi-\xi_{1}\right|+\left|\eta-\eta_{1}\right|\right) .
\end{gathered}
$$


HHJ) $J_{k}: \mathfrak{R} \rightarrow \mathfrak{R}, k=1,2, \ldots, n$ and there exist $e_{k}>0, k=1,2, \ldots, n$ s.t.

$$
\left|J_{k}(\xi)-J_{k}\left(\xi_{1}\right)\right| \leq e_{k}\left|\xi-\xi_{1}\right|
$$

In this system, $y(x, t)$ represents the temperature at the point $x \in \Omega$ at time $t$, the condition $C 3$ represents the temperature at the histories time $t \in[-m l, 0]$. The condition $C 4$ represents the temperature on the boundary $\partial \Omega$ that is equal to zero. The input function $f$ means an external heat sources. In this case, the function $f$ depend on the histories data $y(x, t-l), \ldots, y(x, t-m l)$, which is impacted from the initial delay function $\varphi(x, t)$ for $t \in[-m l, 0]$ in the condition $C 3$. Furthermore, the system is regulated by the regulator $u$ with the sensor mapping $\int_{\Omega} \mathcal{B}(x, \xi) u(\xi, t) d \xi$. Given an admissible regulator set $\mathcal{A}_{a d}=\mathcal{L}_{q}(\Omega \times I)$. We solve the optimization regulation problem $\left(P_{0}\right)$ with the objective functional;

$$
\mathcal{P}(u)=\int_{0}^{T} \int_{\Omega}|y(\xi, t)|^{2}+\|y(\xi, t-l)\|^{2}+\ldots+\|y(\xi, t-m l)\|^{2} d \xi d t+\int_{0}^{T} \int_{\Omega}|u(\xi, t)|^{2} d \xi d t+g(y(x, T)),
$$

where $g \in C\left(\mathfrak{R}, \mathfrak{R}^{+}\right)$. That is, find $u_{0} \in \mathcal{A}_{a d}$ that minimize the objective functional. Let $\mathcal{X}=\mathcal{L}_{p}(\Omega)$. For $t \in[-m l, T]$, define $y(t): \Omega \rightarrow \mathcal{X}$ by

$$
y(t)(x)=y(x, t) \text { for all } x \in \Omega, \quad \text { and } \quad D_{t}^{\alpha} y(t) x=\frac{\partial^{\alpha} y(x, t)}{\partial t^{\alpha}}, \text { for all } y \in X, \quad x \in \Omega .
$$

We define

$$
\begin{gathered}
f(t, y(t), y(t-l), \ldots, y(t-m l))(x)=f(x, t, y(x, t), y(x, t-l), \ldots, y(x, t-m l)), \\
\mathcal{B}(t) u(t)(x)=\int_{\Omega} \mathcal{B}(x, \xi) u(\xi, t) d \xi \text { and } J_{k}(y(t))(x)=J_{k}(y(x, t)) .
\end{gathered}
$$

Define an operator $A: \mathcal{X} \rightarrow \mathcal{X}$ as

$$
A y=\Delta y \text { for all } y \in D(A)
$$

which $D(A)$ contains all $C^{2}(\bar{\Omega})$ function vanishing on $\partial \Omega$.

Now we introduce the eigenvalue problem for the negative Laplacian;

$$
A y=\lambda y \text { for all } y \in D(A)
$$

Using the standard definition of the inner product, we define that for any $y_{1}, y_{2} \in D(A)$;

$$
\left\langle A y_{1}, y_{2}\right\rangle=\int_{\Omega} \overline{y_{2}} \Delta y_{1} d y=\int_{\Omega} \overline{y_{1}} \Delta y_{2} d y=\left\langle y_{1}, A y_{2}\right\rangle .
$$

Therefore, $A$ is symmetric and its eigenvalues must be real. Furthermore, for any $y \in D(A)$, we have

$$
\langle A y, y\rangle=\langle\Delta y, y\rangle=\int_{\Omega} \bar{y} \Delta y d y=\int_{\Omega}|\operatorname{grad} y|^{2} d y \geq 0 .
$$

The right hand side vanishes only if $y$ is constant but the only constant in $D(A)$ is the zero constant. Thus, we obtain

$$
\lambda\|y\|^{2}=\langle\lambda y, y\rangle=\langle A y, y\rangle>0, \text { for all } y \neq 0 \text { in } D(A) .
$$

This is precisely the definition of a positive operator, $A$ is actually strongly positive. Because of Equation $C 5$, the eigenvalues of $A$ must be positive and we obtain a following lemma.

Lemma 13 The operator $A$ is a infinitesimal generator of a compact $C_{0}$-semigroup on $X$.

Applying Lemma 13 , so the system $C 1-C 4$ can transform to the abstract form;

$$
\left\{\begin{array}{l}
D_{t}^{\alpha} y(t)=A y(t)+f(t, y(t), y(t-l), \ldots, y(t-m l))+\mathcal{B}(t) u(t), \quad t \in I \backslash \mathcal{D} \\
\Delta y\left(t_{k}\right)=J_{k}\left(y\left(t_{k}\right)\right), \quad t_{k} \in \mathcal{D} \\
y(t)=\varphi(t), \quad t \in[-m l, 0]
\end{array}\right.
$$


Theorem 14 Suppose that the assumptions $(H H f)$ and $(H H J)$ are sattisfied. Then the problem $\left(P_{0}\right)$ for the nonlinear fractional heat equation with delay in $\mathfrak{R}^{N}, C 1-C 4$ has at least one solution.

Proof. We solve the regulation problem $\left(P_{0}\right)$ for system $C 1-C 4$ pass the abstract form, $C 6$. The definitions of $f, J_{k}(k=1,2, \ldots, n)$ and the objective functional $\mathcal{P}$ and the assumptions (HHf), (HHJ) imply the assumptions of Theorem 11 and Theorem 12. Therefore, the problem $\left(P_{0}\right)$ with system $C 1-C 4$ has at least one solution.

\section{Conclusions}

In this work, we considered the fractional nonlinear differential system (1) with time lag, when $A$ is the infinitesimal generator of a compact semigroup $\{S(t)\}_{t \geq 0}$ satisfying the exponential stability. We proved the existence solution and solved the optimal regulation problem. We proposed a method for proving existence whose main component is the use of the Leray-Schauder Fixed Point Theorem(LSFPT). More precisely, we assume that the input function, $f$ and operator $J_{k}, k \in\{1,2, \ldots, n\}$ satisfy the condition A1-A5. We successfully applied this method and use these assumptions to prove the existence of PC-mild solution. For studying the optimization regulation problem, we win to prove that system (1) has atleast one optimal regulator with conditions A1-A10. Beside the study of the solution and the optimization regulation problem, we give some examples (model of problem in the real world), we give example of $f$ and $J_{k}, k \in\{1,2, \ldots, n\}$ such that satisfying the conditions (HHf) and (HHJ). Then we transform them to the abstract form and use our main results to conclude that these systems have atleast one optimal regulator.

Last but not least we should be interested in developing this method and use weakly assumptions to prove the existence and uniqueness of PC-mild solution a little further. Moreover, we should be interested in studying the others the solution behaviours for example;the stable property. Even though it seems likely that efforts in this direction can be successful, there no guarantee for that. Therefore, we can only hope for the best, but have to expect the worst.

\section{Acknowledgements}

Beside the efforts I put in writing up this research, many people have involved in this project and contributed to its success. I would like to thanks my wife; she encouraged me all of the time and found many data for me. I would like to thanks the money-supported from Faculty of Science of King Mongkut's Institute of Technology Ladkrabang.

\section{References}

Benchohra, M., Henderson, J., Ntouyas, S. K., \& Ouahab, A. (2008). Existence results for fractional order functional differential equations with infinite delay. Applied Mathematical Sciences, 338(2), 1340-1350. http://dx.doi.org/10.1016/j.jmaa.2007.06.021

Diethelm, K., \& Ford, N. J. (2002). Analysis of Fractional Differential Equations. Journal of Mathematical Analysis and Applications, 265(2), 229-248.

Frederico, G. S. F., \& Torres, D. F. M. (2008). Fractional optimal control in the sense of Caputo and the Fractional Noether's theorem. International Mathematical Forum, 3(10), 479-493.

Jumarie, G. (2007). Fractional Partial Differential Equations and Modified Riemann-Liouville Derivative new Methods for Solution. J. Appl. Math and Computing, 24(1-2), 31-48. http://dx.doi.org/10.1007/BF02832299

Lakshmikantham, V., Binov, D. D., \& Simenov, P. S. (1989). Theory of Impulsive Differential Equations. Singapore: World Scientific.

Mainardi, F. (1996). Fractional relaxation-oscillation and fractional diffusion-wave Phenomena. Chaos, Solutions and Fractals, 9, 1461-1477. http://dx.doi.org/10.1016/0960-0779(95)00125-5

McBride, A. C. (1987). Semigroups of Linear Operators: An introduction. Harlow, U.K.: Longman.

Miller, K. S., \& Ross, B. (1993). An Introduction to the Fractional Calculus and Fractional Differential Equations. New York: Willey.

Mophou, G. M. (2009). Existence and uniqueness of mild solution to impulsive fractional differential equations. Nonlinear Analysis, 72(3-4), 1604-1615. http://dx.doi.org/10.1016/j.na.2009.08.046

Pazy, A. (1983). Semigroups of Linear Operators and Applications to Partial Differential Equations. New York: Springer.

Podlubny, I. (2002). Geometric and physical interpretation of fractional integration and fractional differentiation. 
Fractional Calculus and Applied Analysis, 5(4), 367-386.

Rahimy, M. (2010). Applications of Fractional Differential Equations. Applied Mathematical Sciences, 4(50), 2453-2461.

Wei, Z. L., Li, Q. D., \& Che, J. L. (2010). Initial value problems for fractional differential equations involving Riemann-Liouville sequential fractional derivative. Applied Mathematical Sciences, 367(1), $260-272$. http://dx.doi.org/10.1016/j.jmaa.2010.01.023 